\title{
Estrutura e resultados do controle da sífilis em gestantes na atenção básica: estudo transversal
}

\author{
Structure and outcomes of syphilis control in pregnant women in primary care: a cross-sectional study
}

Estructura y resultados del control de la sífilis en embarazadas en atención primaria: un estudio transversal

\author{
Maria Gercileide de Araújo Leal' @ , Edilma Gomes Rocha Cavalcante ${ }^{\prime \prime}$, Emiliana Bezerra Gomes ${ }^{\prime \prime} \odot$, \\ Maria Lúcia Duarte Pereira ${ }^{\prime \prime \prime} \odot$, Rachel de Sá Barreto Luna Callou Cruz" ${ }^{\circledR}$, Dayanne Rakelly de Oliveira ${ }^{\prime \prime}($ \\ 'Prefeitura do Município de Juazeiro do Norte, Juazeiro do Norte, CE Brasil; "Universidade Regional do Cariri, Crato, CE, Brasil; \\ "'Universidade Estadual do Ceará, Fortaleza, Brasil
}

\begin{abstract}
RESUMO
Objetivo: avaliar o serviço de atenção básica quanto à estrutura e aos resultados relativos ao controle de casos de sífilis em gestantes. Método: estudo descritivo de abordagem quantitativa, realizado em unidades básicas de saúde de um município do interior do Ceará, entre março e maio de 2019. Dados analisados por meio de estatística descritiva e inferencial (teste t-student). Resultados: na avaliação global da estrutura, as 43 unidades de saúde foram classificadas como satisfatórias $(p=0,00)$, assim como para os recursos humanos $(p=0,00)$, apoio diagnóstico $(p=0,00)$, organização e registro $(p=0,00)$. Constataram-se limitações quanto à falta de penicilina nas unidades de saúde e nos resultados identificados, $18,9 \%$ na taxa de detecção de sífilis em gestantes e 18,1\% na taxa de incidência de sífilis congênita. Conclusão: a avaliação do controle da sífilis em gestantes em 2018 apontou que, no componente estrutura, faltam penicilina benzatina e capacitação profissional sobre anafilaxia. Os casos de sífilis em gestantes e congênita estão acima dos níveis nacionais.
\end{abstract}

Descritores: Atenção Primária à Saúde; Cuidado Pré-Natal; Sífilis; Avaliação de Resultados em Cuidados de Saúde.

\section{ABSTRACT}

Objective: to evaluate the primary care service as to structure for, and outcomes in, control of syphilis cases in pregnant women. Method: this quantitative, descriptive study was conducted in basic health units in a city in Ceará state, between March and May 2019. The data were analyzed using descriptive and inferential statistics (Student's t-test). Results: the 43 health units were classified as satisfactory in overall structure, $(p=0.00)$, human resources $(p=0.00)$, diagnostic support $(p=0.00)$, and organization and recording $(p=0.00)$. Restrictions were found as regards the lack of penicillin in the health unities, being observed in $18.9 \%$ of the detection rate of syphilis in pregnant women and $18.1 \%$ of the incidence rate of congenital syphilis. Conclusion: evaluation of syphilis control in pregnant women in 2018 indicated, in the structure component, a lack of benzathine penicillin and professional training in anaphylaxis. Cases of syphilis in pregnant women and congenital syphilis are above national levels.

Descriptors: Primary Health Care; Prenatal Care; Syphilis; Outcome Assessment, Health Care.

\section{RESUMEN}

Objetivo: evaluar el servicio de atención primaria en cuanto a la estructura y los resultados relacionados con el control de casos de sífilis en embarazadas. Método: estudio descriptivo con enfoque cuantitativo, realizado en unidades básicas de salud de una ciudad del interior de Ceará, realizado entre marzo y mayo de 2019. Se analizaron los datos mediante estadística descriptiva e inferencial (test t-student). Resultados: en la evaluación global de la estructura, se clasificaron las 43 unidades de salud como siendo satisfactorias $(p=0,00)$, así como en cuanto a recursos humanos $(p=0,00)$, apoyo diagnóstico $(p=0,00)$, organización y registro ( $p$ $=0,00$ ). Se verificaron limitaciones respecto a la falta de penicilina en los establecimientos de salud y en los resultados identificados, $18,9 \%$ en la tasa de detección de sífilis en embarazadas y $18,1 \%$ en la tasa de incidencia de sífilis congénita. Conclusión: La evaluación del control de la sífilis en embarazadas en 2018 indicó que, en el componente estructural, faltan penicilina benzatínica y formación profesional en anafilaxia. Los casos de sífilis en embarazadas y congénitas están por encima de los niveles nacionales. Descriptores: Atención Primaria de Salud; Atención Prenatal; Sífilis; Evaluación de Resultado en la Atención de Salud.

\section{INTRODUÇÃO}

A sífilis é um grave problema de saúde pública quando acomete gestantes e causa abortamento, prematuridade, mortalidade neonatal e manifestações congênitas ${ }^{1}$. Tem-se observado um aumento de casos de sífilis em gestante e sifilis congênita nos últimos anos, sendo a meta para eliminação desse último de 0,5 caso por 1.000 nascidos vivos por ano.

Em 2018, no Brasil, foram 62.599 casos de sífilis em gestantes com taxa de incidência de 9,0/1.000 nascidos vivos². Enquanto, no Ceará, o município de Juazeiro do Norte notificou 65 casos de sífilis em gestantes e 47 de sífilis congênita ${ }^{3,4}$.

Dentre os desafios para o controle de tratamento da sífilis em gestantes e sífilis congênita, estão a estrutura e a organização dos serviços da atenção básica, pois se identifica que, ao aumentar os percentuais de equipes de saúde com estrutura e oferta de deteç̧ão e tratamento de casos, é possível manter a relação entre as ações e a redução de sífilis congênita 5 . 
Além do mais, considera-se que a assistência ao pré-natal, mesmo contribuindo para a detecção precoce dos casos com a disponibilização de testes rápidos, não tem gerado impacto na redução da taxa de incidência de sífilis. Diante de tal fato, identificam-se limitações que devem ser alvo de intervenções para promover a prevenção e o bloqueio da transmissão vertical da doença ${ }^{6}$.

Nesse contexto, os profissionais de saúde devem realizar ações que assegurem a qualidade da atenção à gestante e ao recém-nascido no que tange o manejo da infecção por sífilis ${ }^{7}$, bem como reforçar a detecção precoce, o manejo adequado dos casos (mulher e parceiro) e a conscientização do uso de preservativo para a população em geral ${ }^{5}$. Além disso, é importante aumentar a cobertura de testes diagnósticos, capacitar profissionais de saúde sobre diagnóstico, tratamento e acompanhamento da sífilis ${ }^{8}$.

Diante do exposto, emergiu o seguinte questionamento: como se encontra a estrutura e os resultados do controle de casos de sífilis em gestantes na atenção básica? Este estudo objetivou avaliar o serviço de atenção básica quanto à estrutura e aos resultados relativos ao controle de casos de sífilis em gestantes.

\section{MÉTODO}

Estudo transversal e de abordagem quantitativa, que utilizou componentes relacionados à estrutura e resultado ${ }^{9}$ de 43 Unidades Básicas de Saúde (UBS) de um município do Sul do Ceará, o que representa 89,6\% de UBS distribuídas em oito distritos sanitários, os quais contavam com quatro a nove UBS.

Foram critérios de inclusão: ter, pelo menos, uma Equipe de Saúde da Família cadastrada; e ser a UBS sede dessa equipe. Foram excluídas as UBS que estavam fechadas, em reforma ou sem funcionamento no período da coleta de dados.

A pesquisa aconteceu entre março e maio de 2019, sendo desenvolvida em duas etapas, a saber: a) Avaliação da Estrutura: coleta única, após agendamento prévio com o enfermeiro gerente da UBS, que auxiliou no levantamento dos itens relacionados à estrutura, como: consultórios, salas de procedimentos, sala de triagem, farmácia, sala de imunização, sala de educação em saúde e outros; e b) Avaliação dos Resultados: a partir de dados coletados no Sistema de Informação de Agravos e Notificação (SINAN) e no Sistema de Nascidos Vivos (SINASC), disponibilizados pelo Setor de Vigilância à Saúde do Município.

Para a análise da estrutura, elaborou-se um cheklist com base nas normas técnicas do Manual da Estrutura Física das UBS, dispostas na Portaria no 3.161/2011 sobre a administração da penicilina nas UBS, e no instrumento de Avaliação da Saúde do Programa Nacional de Melhoria do Acesso e da Qualidade da Atenção Básica $(P M A Q)^{10,11,12}$. Ressalta-se que foi realizado um teste piloto para verificar a adequabilidade do instrumento de coleta de dados, mas não houve necessidade de modificação.

Assim, o checklist era composto por 52 questões sobre estrutura física, recursos humanos e materiais, apoio diagnóstico, medicamentos e processo organizacional e de registro, sendo que os itens de cada variável continham as seguintes classificações: insatisfatório ou inexistente; parcialmente atendidas; e totalmente atendidas, com as atribuições de zero, cinco e dez pontos, respectivamente ${ }^{12,13}$. Neste sentido, a soma de pontos de cada variável totaliza pontuações máximas distintas (planta física - 60 pontos; recursos humanos - 60 pontos; recursos materiais - 240 pontos; apoio diagnóstico - 60 pontos; medicamentos - 50 pontos; e processo organizacional e de registro 50 pontos), de modo que as 52 questões acerca dos componentes da estrutura da UBS perfazem o máximo de 520 pontos.

Pondera-se que cada categoria foi analisada individualmente com a sua pontuação e respectiva média atingida. O percentual alcançado foi classificado em ótima ( $90,0 \%$ a $100,0 \%$ do total de pontos), satisfatória (75,0\% a $89,9 \%)$, precária $(50,0 \%$ a $74,9 \%)$ ou insuficiente $(49,9 \% \text { e menos })^{13,14}$.

Para a análise dos resultados, utilizaram-se os dados dos nascidos vivos do SINASC e o número de casos de sífilis em gestante e congênita do SINAN, no ano de 2018. A taxa de detecção de sífilis em gestante foi calculada pelo número de casos notificados divididos pelo número de nascidos vivos, multiplicado por 1.000. A taxa de detecção de sífilis congênita foi feita pelo número total de casos notificados de sífilis congênita em menores de um ano de idade dividido pelo número total de nascidos vivos, multiplicado por 1.000 .

Utilizou-se o programa estatístico Statistical PacKage for the Social Sciences (IBM SPSS ${ }^{\circledR}$ ), versão 20.0, analisado por meio de estatística descritiva (números absolutos e relativos, medidas de tendência central e dispersão) e inferencial pela comparação de médias dos itens com a adequação (teste $t$-student), considerado significativo $(p<0,05)$ e apresentado em tabelas.

O estudo foi aprovado pelo Comitê de Ética em Pesquisa com Seres Humanos da instituição e respeitou todos os preceitos éticos da pesquisa que envolve seres humanos. 


\section{RESULTADOS}

Identificou-se que o percentual máximo de pontos (520) referentes à estrutura, foram atendidos por apenas duas UBS, avaliadas como ótimas (> $90,0 \%$ dos pontos), com 33 classificadas como satisfatórias (75,0\% a 89,0\% pontos) e oito como precárias (50,0\% a 74,9\% pontos). Observou-se que apenas o Distrito VI contava com UBS consideradas ótimas; no Distrito V, todas eram satisfatórias e, entre 6 Distritos, identificou-se pelo menos uma UBS considerada em condições precárias.

Na Tabela 1, verifica-se que na avaliação global da estrutura.

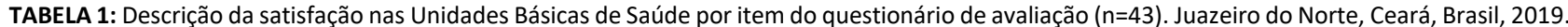

\begin{tabular}{|c|c|c|c|c|}
\hline Variáveis & Insatisfatório & Parcialmente satisfatório & Totalmente satisfatório & p-valor* / (X+DP) \\
\hline Estrutura física & & & & $0,00 * /(8,565 \pm 1,784)$ \\
\hline Consultório & - & $1(2,3 \%)$ & $42(97,7 \%)$ & \\
\hline Farmácia & - & $1(2,3 \%)$ & $42(97,7 \%)$ & \\
\hline Pia no consultório & $2(4.7 \%)$ & $5(11,6 \%)$ & $36(83,7 \%)$ & \\
\hline $\begin{array}{l}\text { Sala de administração de } \\
\text { medicamentos }\end{array}$ & $6(14,0 \%)$ & $6(14,0 \% 0)$ & $31(72,0 \%)$ & \\
\hline Sala educação saúde & $11(25,6 \%)$ & - & $32(74,4 \%)$ & \\
\hline Acessibilidade & $6(14,0 \%)$ & $11(25,6 \%)$ & $26(60,4 \%)$ & \\
\hline Recursos humanos & & & & $0,00 * /(7,538 \pm 1,177)$ \\
\hline Pré-natal $1 x /$ sem & - & - & $43(100,0 \%)$ & \\
\hline Médico e Enfermeiro no pré-natal & - & $22(51,2 \%)$ & $21(48,8 \%)$ & \\
\hline ACS & & $16(37,2 \%)$ & $27(62,8 \%)$ & \\
\hline Técnico de Enfermagem & & & $43(100,0 \%)$ & \\
\hline Capacitação em sífilis & $4(9,3 \%)$ & $11(25,3 \%)$ & $28(65,1 \%)$ & \\
\hline Capacitação em anafilaxia & $32(74,4 \%)$ & $5(11,6 \%)$ & $6(14,0 \%)$ & \\
\hline Recursos materiais & & & & $0,00 * /(8,929 \pm 0,535)$ \\
\hline Mesa ginecológica & - & $1(2,3 \%)$ & $42(97,7 \%)$ & \\
\hline Foco de luz & - & - & $43(100,0 \%)$ & \\
\hline Balança adulto & - & - & $43(100,0 \%)$ & \\
\hline Esfigmomanômetro & $1(2,3 \%)$ & $3(7,0 \%)$ & $39(90,7 \%)$ & \\
\hline Termômetro clínico & - & - & $43(100,0 \%)$ & \\
\hline Estetoscópio & - & - & $43(100,0 \%)$ & \\
\hline Sonar & - & $3(7,0 \%)$ & $40(93,0 \%)$ & \\
\hline Fita métrica & - & - & $43(100,0 \%)$ & \\
\hline Espéculos & - & - & $43(100,0 \%)$ & \\
\hline Pinça cheron & $1(2,3 \%)$ & - & $42(97,7 \%)$ & \\
\hline Espátula de ayre & - & - & $43(100,0 \%)$ & \\
\hline Escova endocervical & - & - & $43(100,0 \%)$ & \\
\hline Lugol & - & - & $43(100,0 \%)$ & \\
\hline Ácido acético & - & - & $43(100,0 \%)$ & \\
\hline Lixeira com pedal & $1(2,3 \%)$ & $4(9,3 \%)$ & $38(88,4 \%)$ & \\
\hline Descartex & $1(2,3 \%)$ & $22(51,2 \%)$ & $20(46,5 \%)$ & \\
\hline Seringa $3 \mathrm{ml}$ & - & - & $43(100,0 \%)$ & \\
\hline Seringa $5 \mathrm{ml}$ & $5(11,7 \%)$ & $1(2,3 \%)$ & $37(86 \%)$ & \\
\hline Seringa $10 \mathrm{ml}$ & $4(9,3 \%)$ & $1(2.3 \%)$ & $38(88,4 \%)$ & \\
\hline Luva procedimento & - & - & $43(100 \%)$ & \\
\hline Preservativo masculino & $7(16,3 \%)$ & $3(7,0 \%)$ & $33(76,7 \%)$ & \\
\hline Preservativo feminino & $20(46,5 \%)$ & $3(7,0 \%)$ & $20(46,5 \%)$ & \\
\hline Manual de sífilis ou IST & $22(51,2 \%)$ & & $21(48,8 \%)$ & \\
\hline Material educativo sífilis & $27(62,7 \%)$ & $2(4,7 \%)$ & $14(32,6 \%)$ & \\
\hline Apoio diagnóstico & & & & $0,00 * /(8,934 \pm 1,210)$ \\
\hline USG obstétrica & $2(4,7 \%)$ & $22(51,1 \%)$ & $19(44,2 \%)$ & \\
\hline Papanicolau & - & $5(11,6 \%)$ & $38(88,4 \%)$ & \\
\hline Teste Hep. B & $1(2,3 \%)$ & $1(2,3 \%)$ & $41(95,4 \%)$ & \\
\hline Teste rápido HIV & $1(2,3 \%)$ & $1(2,3 \%)$ & $41(95,4 \%)$ & \\
\hline VDRL & - & $7(16,3 \%)$ & $36(83,7 \%)$ & \\
\hline Teste rápido de sífilis & $5(11,6 \%)$ & $1(2,3 \%)$ & $37(86,0 \%)$ & \\
\hline
\end{tabular}

\footnotetext{
*Teste t-student.
} 
TABELA 1: Descrição da satisfação nas Unidades Básicas de Saúde por item do questionário de avaliação ( $n=43$ ). Juazeiro do Norte, Ceará, Brasil, 2019 (continuação).

\begin{tabular}{|c|c|c|c|c|}
\hline Variáveis & Insatisfatório & Parcialmente satisfatório & Totalmente satisfatório & $\overline{p-v a l o r * /(X \pm D P)}$ \\
\hline Medicamentos & & & & $0,00 * /(2,883 \pm 1,854)$ \\
\hline Adrenalina & $37(86,0 \%)$ & - & $6(14,0 \%)$ & \\
\hline Soro fisiológico & $1(2.3 \%)$ & $1(2.3 \%)$ & $41(95,4 \%)$ & \\
\hline Fenergan & $38(88,4 \%)$ & - & $5(11,0 \%)$ & \\
\hline Penicilina Benzatina & $43(100,0 \%)$ & - & - & \\
\hline Água destilada & $33(76,7 \%)$ & $1(2,3 \%)$ & $9(20,9 \%)$ & \\
\hline Processo organizacional e registro & & & & $0,00 * /(7,627 \pm 1,380)$ \\
\hline Referência & $1(2,3 \%)$ & - & $42(97,7 \%)$ & \\
\hline Contrarreferência & $32(74.4 \%)$ & $5(11,6 \%)$ & $6(14,0 \%)$ & \\
\hline Cartãogestante & $1(2,3 \%)$ & $2(4,7 \%) i$ & $40(93,0 \%)$ & \\
\hline Ficha perinatal & - & $1(2,3 \%)$ & $42(97,7 \%)$ & \\
\hline Ficha notificação Sífilis em gestante & $12(27,9 \%)$ & $2(4,7 \%)$ & $29(67,4 \%)$ & \\
\hline
\end{tabular}

*Teste t-student.

Observa-se que 43 UBS eram satisfatórias (média $=85,6 \% ; p=0,00$ ). Todas dispunham de pré-natal pelo menos uma vez por semana. Os recursos humanos foram satisfatórios (média $=75,5 \%, p: 0,00$ ), sendo parcialmente satisfatória a presença de médicos e enfermeiros (51,2\%); totalmente satisfatória a capacitação em sífilis nos últimos dois anos $(65,1 \%)$ e insatisfatória em anafilaxia $(74,4 \%)$. Os recursos materiais foram satisfatórios (média $=89,2, p=0,00$ ) com ausência do preservativo feminino (46,5\%), manual de sífilis ou IST (48,8\%) e material educativo de sífilis/IST (62,7\%).

O apoio diagnóstico foi satisfatório (média $=89,3 \%, p=0,00$ ) com testes rápidos em quantidade suficiente (95,3\%), visto que o VDRL era parcialmente disponibilizado em 7 UBS (16,3\%). A ultrassonografia (USG) obstétrica foi parcialmente satisfatória (51,1\%). Os medicamentos foram insuficientes (média $=28,8 \%, p=0,00$ ) e nenhuma UBS possuía Penicilina Benzatina. $O$ processo organizacional e de registro era satisfatório (média $=76,2, p=0,00$ ), especialmente quanto à presença de ficha de notificação/investigação de sífilis em gestantes em quantidade suficiente $(67,4 \%$;).

$\mathrm{Na}$ Tabela 2, identifica-se que não houve significância estatística na associação entre os itens da estrutura avaliados referentes ao diagnóstico e tratamento de sífilis, mas apresentaram certa homogeneidade entre a adequação e inadequação da capacitação dos profissionais para o acompanhamento dos casos de sífilis.

TABELA 2: Descrição da avaliação da estrutura relacionada aos diagnósticos e tratamento dos casos de sífilis acompanhando quanto à satisfação. Juazeiro do Norte, Ceará, Brasil, 2019.

\begin{tabular}{|c|c|c|c|c|}
\hline \multirow{2}{*}{\multicolumn{2}{|c|}{$\begin{array}{c}\text { Variáveis de estrutura relacionadas } \\
\text { ao diagnóstico e tratamento }\end{array}$}} & \multicolumn{3}{|c|}{ Treinamento para acompanhamento dos casos de sífilis } \\
\hline & & Insatisfatório & Satisfatório & p-valor* \\
\hline \multirow{2}{*}{ Teste rápido HIV } & Inadequado & $0(0,0 \%)$ & $2(100,0 \%)$ & \multirow{2}{*}{0,535} \\
\hline & Adequado & $15(36,6 \%)$ & $26(63,4 \%)$ & \\
\hline \multirow{2}{*}{ VDRL } & Inadequado & $1(14,3 \%)$ & $6(85,7 \%)$ & \multirow[t]{2}{*}{0,391} \\
\hline & Adequado & $14(38,9 \%)$ & $22(61,1 \%)$ & \\
\hline \multirow{2}{*}{ Teste rápido de sífilis } & Inadequado & $2(33,3 \%)$ & $4(66,7 \%)$ & \multirow{2}{*}{1,000} \\
\hline & Adequado & $13(35,1 \%)$ & $24(64,9 \%)$ & \\
\hline \multirow{2}{*}{ Penicilina Benzatina } & Inadequado & - & - & \multirow[t]{2}{*}{-} \\
\hline & Adequado & $15(34,9 \%)$ & $28(65,1 \%)$ & \\
\hline \multirow{2}{*}{ Água destilada } & Inadequado & $12(35,3 \%)$ & $22(64,7 \%)$ & \multirow{2}{*}{1,000} \\
\hline & Adequado & $3(33,3 \%)$ & $6(66,7 \%)$ & \\
\hline \multirow{2}{*}{ Referência } & Inadequado & $1(100,0 \%)$ & $0(0,0 \%)$ & \multirow{2}{*}{0,349} \\
\hline & Adequado & $14(33,3 \%)$ & $28(66,7 \%)$ & \\
\hline \multirow{2}{*}{ Contrarreferência } & Inadequado & $13(35,1 \%)$ & $24(64,9 \%)$ & \multirow{2}{*}{1,000} \\
\hline & Adequado & $2(33,3 \%)$ & $4(66,7 \%)$ & \\
\hline \multirow{2}{*}{ Cartão gestante } & Inadequado & $1(33,3 \%)$ & $2(66,7 \%)$ & \multirow{2}{*}{1,000} \\
\hline & Adequado & $14(35,0 \%)$ & $26(65,0 \%)$ & \\
\hline \multirow{2}{*}{ Ficha perinatal } & Inadequado & $1(100,0 \%)$ & $0(0,0 \%)$ & \multirow{2}{*}{0,349} \\
\hline & Adequado & $14(33,3 \%)$ & $28(66,7 \%)$ & \\
\hline \multirow{2}{*}{ Ficha notificação sífilis em gestante } & Inadequado & $3(21,4 \%)$ & $11(78,6 \%)$ & \multirow{2}{*}{0,308} \\
\hline & Adequado & $12(41,4 \%)$ & $17(58,6 \%)$ & \\
\hline
\end{tabular}

*Teste Exato de Fisher 
A Tabela 3 apresenta os casos absolutos e indicadores de sífilis em gestantes e congênita.

Tabela 3: Variável de avaliação de resultado de sífilis em gestantes e congênita. Juazeiro do Norte, Ceará, Brasil, 2018.

\begin{tabular}{lcr}
\hline Variáveis & $\mathbf{n}$ & $\mathbf{f ( \% )}$ \\
\hline Casos notificados de sífilis em gestantes & 80 & \\
Casos notificados de sífilis congênita & 77 & \\
Casos de sífilis notificados nas UBS & 0 & \\
Casos de nascidos vivos & 4238 & \\
Taxa de detecção de sífilis em gestantes & & 18,9 \\
Taxa de incidência de sífilis congênita & & 18,1 \\
\hline Fonte: Secretaria Municipal de Saúde de Juazeiro do Norte (2018).
\end{tabular}

Nenhum caso foi notificado na UBS, em 2018 , com 18,9\% na taxa de detecção de sífilis em gestantes e 18,1\% na taxa de incidência de sífilis congênita.

\section{DISCUSSÃO}

Na avaliação do serviço de atenção básica, identificou-se um resultado controverso em relação à adequabilidade da estrutura na maioria das UBS, no entanto, as taxas de detecção de sífilis em gestante e sífilis congênita se encontram acima da média nacional.

$\mathrm{Na}$ avaliação global da estrutura das UBS, verificou-se que a maioria é satisfatória quanto à média de itens. Os recursos humanos são classificados como satisfatórios no atendimento às gestantes, mas parcialmente satisfatórios quanto à presença de profissionais de nível superior, em especial de médicos. Este resultado se assemelha ao encontrado em um estudo ecológico que aponta a relação entre o maior percentual de equipes de atenção básica, a oferta de tratamento com penicilina e a redução da incidência de sífilis gestacional ${ }^{14}$.

No presente estudo, constatou-se que a capacitação profissional sobre a abordagem da sífilis é satisfatória, mas não em relação à anafilaxia. No Brasil, os profissionais de saúde apresentam conhecimentos inadequados ou insuficientes sobre o manejo da sífilis na gravidez ${ }^{7,15}$. Já na Colômbia, os obstáculos para a condução do pré-natal se relacionam com a falta de atualização acerca do diagnóstico e tratamento da sífilis, bem como da baixa disponibilização de testes rápidos para IST e penicilina benzatina nos serviços de saúde ${ }^{15,16}$. Todavia, apesar da importância de capacitações para as equipes, as instituições devem dispor de estratégias para operacionalizar o tratamento imediato diante de casos de sífilis em gestantes, condição que deve ser revista diante da realidade epidemiológica local.

A adequação dos insumos para diagnóstico e tratamento oportuno deve ser garantida nas UBS, a fim de evitar negligências no acompanhamento de gestante, que têm como consequências o diagnóstico tardio de sífilis e, consequentemente, a transmissão vertical. Sobre este aspecto, esta pesquisa encontrou que os recursos materiais são satisfatórios, assim como o apoio diagnóstico relativo aos testes rápidos, com menor disponibilidade para de VDRL.

À despeito da importância dos testes rápidos para o manejo da doença durante o pré-natal, ou em qualquer oportunidade de atendimento à mulher e seus parceiros ${ }^{7,17}$, constata-se um maior percentual de equipes da atenção básica que ofertam testes rápidos ${ }^{5,18}$, mas um pouco mais da metade das gestantes realizam exames no início do terceiro trimestre e $57 \%$ dos parceiros não foram testados ${ }^{18}$.

Em relação aos medicamentos, estes se mostram insuficientes pela ausência de Penicilina Benzatina, o que concorda com resultados de outros estudos que revelam sua indisponibilidade no âmbito da atenção básica, apesar da administração dessa medicação neste âmbito assistencial ser uma recomendação, frente às altas taxas de incidência da sífilis em gestantes ${ }^{18,19}$. Por outro lado, pesquisa realizada em um estado da Região Nordeste revelou que esta medicação está disponível em 87,1\% das equipes da Estratégia Saúde da Família, porém, quase $50 \%$ delas não a administram ${ }^{18}$ e conduzem as gestantes ao ambiente hospitalar diante da falta de insumos para manejar casos de reação anafilática ${ }^{20}$.

Neste estudo, constatou-se que os profissionais de saúde são capacitados para o atendimento à sífilis, mas as notificações das gestantes têm ocorrido no momento do parto em ambiente hospitalar. Um estudo de Londrina observou que grande parte das mulheres foi diagnosticada durante o pré-natal, a maioria entre o segundo e o terceiro trimestres de gravidez ${ }^{15}$. Em contrapartida, na Colômbia, dentre os 316 casos notificados de sífilis gestacional, $80 \%$ foi no pré-natal e $20 \%$ no momento do parto ${ }^{21}$. Diante do exposto, ressalta-se que os serviços das UBS devem notificar e tratar os casos ainda no pré-natal, assim como utilizar estratégias que auxiliem o monitoramento dos casos e controle da doença. 
No ano de 2018, praticamente todos os casos de sífilis em gestantes no município estudado resultaram em casos de sífilis congênita e nenhuma notificação foi realizada pelas equipes de saúde da família, achado que se alinha às deficiências estruturais e técnicas identificadas no acompanhamento e na abordagem terapêutica das gestantes. Além das fragilidades da estrutura das unidades de saúde quanto ao tratamento de sífilis em gestantes, também foram constatadas falhas no acompanhamento e na notificação de casos, que resultaram em transmissão vertical.

Corroborando, estudo realizado no município do Fortaleza apontou que as condições das unidades de saúde, envolvendo problemas organizacionais, da estrutura física e da continuidade do atendimento, bem como as dificuldades dos profissionais de saúde no manejo de infecções sexualmente transmissíveis são elementos que interferem na prevenção e controle da sífilis congênita, com impactos sobre o aumento do indicador de casos da doença ${ }^{20}$.Identificase também que a atenção primária à saúde enfrenta desafios relacionados ao seu papel na detecção do diagnóstico precoce da sífilis e no tratamento de gestante e parceiro ${ }^{22}$.

Diante do exposto, parece contraditório que em áreas com cobertura de estratégia de saúde da família, as quais poderiam assegurar o diagnóstico precoce e a assistência oportuna aos casos de gestantes com sífilis, de fato, têm perdido essa oportunidade e não conseguem reduzir a sífilis congênita.

Como limitação deste estudo, ressalta-se a não incorporação do processo de trabalho das equipes de saúde como um elemento da avaliação, que por sua vez poderia esclarecer algumas indefinições, como a operacionalização adequada de alguns componentes da estrutura das UBS.

\section{CONCLUSÃO}

$\mathrm{Na}$ atenção básica à saúde, a avaliação do controle da sífilis em gestantes no ano de 2018 apontou que, sobre o componente estrutura, faltam equipamentos específicos utilizados na assistência, como penicilina benzatina e capacitação profissional sobre anafilaxia. Já o componente resultado apresentou casos de sífilis em gestantes e sífilis congênita acima dos níveis nacionais.

Assim, os resultados deste estudo mostram que é necessário fortalecer a capacidade de resposta da atenção básica no que tange o controle da sífilis em gestantes e a prevenção da transmissão vertical, por meio de ações resolutivas com a notificação e o tratamento imediato das gestantes/parceiros.

Este panorama revela a necessidade de melhorar os indicadores de saúde por meio de estratégias a serem desenvolvidas, tanto pela vigilância epidemiológica quanto pelos profissionais da atenção básica, a fim de assegurar a notificação, o manejo precoce e cuidados efetivos à saúde e ao controle de sífilis na gestação e congênita.

\section{REFERÊNCIAS}

1. Ministério da Saúde (Br). Secretaria de Vigilância em Saúde. Departamento de Doenças de Condições Crônicas e Infeç̧ões Sexualmente Transmissíveis. Protocolo Clínico e Diretrizes Terapêuticas para Atenção Integral às Pessoas com Infecções Sexualmente Transmissíveis (IST). Brasília (Br): Ministério da Saúde; 248 p. 2020. [cited 2021 nov 11]. Available from: http://portaldeboaspraticas.iff.fiocruz.br/wp-content/uploads/2020/08/pcdt_ist_final_revisado_020420.pdf.

2. Ministério da Saúde (Br). Boletim Epidemiológico de Sífilis, Secretaria de Vigilância em Saúde. Número especial. Out. 2019. Brasília (Br): Ministério da Saúde; 2019. [cited 2021 Out 17]. Available from: Avaible from: http://www.aids.gov.br/pt$\mathrm{br} /$ pub/2019/boletim-epidemiologico-sifilis-2019.

3. Secretaria de Saúde do Estado do Ceará (CE). Boletim epidemiológico - Sífilis 19 de outubro de 2017. Secretaria de Saúde do Estado: Fortaleza; 2017. [cited 2020 nov 18]. Available from: http://www.saude.ce.gov.br/wpcontent/uploads/sites/9/2018/06/boletim_epidemiologico_sifilis_18_10_2017.pdf.

4. Secretaria de Saúde do Estado do Ceará (CE). Boletim epidemiológico - Sífilis. Secretaria de Saúde do Estado: Fortaleza; 2018. [cited 2021 Out 17]. Available from: http://www.saude.ce.gov.br/wpcontent/uploads/sites/9/2018/06/boletim_epidemiologico_sifilis_18_10_2017.pdf.

5. Figueiredo DCMM, Figueiredo AM, Souza TKB, Tavares G, Vianna RPT. Relationship between the supply of syphilis diagnosis and treatment in primary care and incidence of gestational and congenital syphilis. Cad Saúde Pública. 2020 [cited 2020 nov 18]; 36(3):e00074519. DOI: https://dx.doi.org/10.1590/0102-311X00074519.

6. Soares MAS, Aquino R. Association between the incidence rates for gestational syphilis and congenital syphilis and prenatal care coverage in the State of Bahia, Brazil. Cad. Saúde Pública [Internet]. 2021 [cited 2021 Out 17]; 37(7):e00209520. DOI: http://dx.doi.org/10.1590/0102-311X00209520.

7. Cardoso ARP, Araújo MAL, Cavalcante MS, Frota MA, Melo SP. Analysis of cases of gestational and congenital syphilis between 2008 and 2010 in Fortaleza, State of Ceará, Brazil. Ciênc saúde coletiva [Internet]. 2018 [cited 2019 Set 18]; 23(2):563-574. DOI: http://dx.doi.org/10.1590/1413-81232018232.01772016.

8. Silveira MF, Leon RGP, Becerra F, Serruya SJ. Evolution towards the elimination of congenital syphilis in Latin America and the Caribbean: a multicountry analysis. Rev Panam Salud Publica [Internet]. 2019 [cited 2020 jan 30]; 43:e31. DOI: http://dx.doi.org/10.26633/RPSP.2019.31.

9. Donabedian, A. La dimensión internacional de La evaluación y garantia de La calidad. Roma: Nuova Italia; 1990. 
10. Ministério da Saúde (Br). Departamento de atenção Básica. Manual de estrutura físicas das unidades básicas de saúde: saúde da família. Série A. Normas e Manuais Técnicos. Brasília; 2008. [cited 2021 Out 17]. Available from: http://bvsms.saude.gov.br/bvs/publicacoes/manual_estrutura_fisica_ubs.pdf.

11. Ministério da Saúde (Br). Portaria № 3.161, de 27 de dezembro de 2011. Dispõe sobre a administração da penicilina nas unidades de Atenção Básica à Saúde, no âmbito do Sistema Único de Saúde (SUS). Brasília (Br): Ministério da Saúde; 2011. [cited 2021 Oct 17]. Available from: http://bvsms.saude.gov.br/bvs/saudelegis/gm/2011/prt3161_27_12_2011.html.

12. Ministério da Saúde (Br). Secretaria de Atenção à Saúde. Departamento de Atenção Básica.Instrumento de Avaliação da Saúde do Saúde mais perto de você- acesso e qualidade. Programa Nacional de Melhoria do Acesso e da Qualidade da Atenção Básica (PMAQ)- Terceiro Ciclo. Brasília (Br): Ministério da Saúde; 2017. [cited 2021 Out 17]. Available from: http://189.28.128.100/dab/docs/portaldab/documentos/Instrumento_Avaliacao_Externa_AB_SB.pdf.

13. Rocha RS, Silva MGC. Prenatal care in the primary health care network in Fortaleza-CE: an assessment of the structure, process and results. Rev. bras. promoç. saúd. [Internet]. 2012 [cited 2020 Set 20]; 25(3):344-55. Available from: https://periodicos.unifor.br/RBPS/article/view/2265.

14. Silveira DS, Santos IS dos, Costa JCS. Prenatal care at the primary health care level: an assessment of the structure and process. Cad Saúde Pública [Internet]. 2001 [cited 2019 Set 20]; 17(1): 131-39. DOI: http://doi.org/10.1590/S0102-311X2001000100013.

15. Lazarini FM, Barbosa DA. Educational intervention in Primary Care for the prevention of congenital syphilis. Rev Latino-Am Enfermagem [Internet]. 2017 [cited 2020 Set 20]; 25:e2845. DOI: http://dx.doi.org/10.1590/1518-8345.1612.2845.

16. Garcés JP, Rubiano LC, Orbio Y, Castaño M, Banavides E, Cruz A. La educación del personal de salud: clave para La eliminación de la sífilis congênita em Colombia. Biomédica on line [Internet]. 2017 [cited 2020 Set 20]; 37(3); 416-24. DOI: http://dx.doi.org/10.7705/biomedica.v37i3.3397.

17. Araújo EC, Monte PCB, Haber ANCA. Evaluation of prenatal care for syphilis and HIV detection in pregnant women attended in a rural area of Pará State, Brazil. Rev Pan-Amaz [Internet]. 2018 [cited 2020 Nov 18]; 9(1):33-9. DOI: http://dx.doi.org/10.5123/s2176-62232018000100005.

18. Araújo TCV, Souza MB. Team adherence to rapid prenatal testing and administration of benzathine penicillin in primary healthcare. Rev Esc Enferm USP. 2020 [cited 2020 Nov 18]; 54:e03645. DOI: https://dx.doi.org/10.1590/S1980$220 \times 2019006203645$.

19. Rodrigues DC, Domingues RMSM. Management of syphilis in pregnancy: Knowledge and practices of health care providers and barriers to the control of disease in Teresina, Brazil. Int J Health Plann Manag [Internet]. 2018 [cited 2020 nov 18]; 33(2): 32944. DOI: https://dx.doi.org/10.1002/hpm.2463.

20. Guanabara MAO, Araújo MAL, Matsue RY, Barros VL, Oliveira FA. Acesso de gestantes às tecnologias para prevenção e controle da sífilis congênita em Fortaleza-Ceará, Brasil. Rev. Salud Pública [Internet]. 2017 [cited 2020 Set 20]; 19(1):73-8. DOI: https://dx.doi.org/10.15446/rsap.v19n1.49295.

21. Loiaza RDA, Arredondo MDC, Rivas CD. Caracterización de la sífilis congénita y gestacional en Caldas, Colombia. Arch Med (Manizales) [Internet]. 2016 [cited 2019 Set 11]; 16(2):326-24. DOI: https://doi.org/10.30554/archmed.16.2.1736.2016.

22. Coleta AVR, Rocha CAWC, Gomes FR, Gouveia G, Oliveira GA, Santana MD et al. Action of primary care in the diagnosis and treatment of syphilis in pregnancy. Brazilian Journal of Health Review [Internet]. 2021 [cited 2021 Nov 11]; 4(5):21165-79. DOI: https://dx.doi.org/10.34119/bjhrv4n5-212. 